\title{
A photoacoustic microscopy system using MEMS and fibre tip transducers for all-optical control
}

\author{
Mark Donnachie*, Peter W. Tinning, Deepak Uttamchandani, Ralf Bauer \\ Centre for Microsystems and Photonics, Department of Electronic \& Electrical Engineering, \\ University of Strathclyde, Glasgow, G1 1XW
}

\begin{abstract}
A 3D photoacoustic microscopy (PAM) system is presented and characterized, using optical MEMS and fibre tip transducers as active elements to provide all-optical positioning and read-out. The excitation beam position is controlled using an electrostatically actuated 2-axis MEMS scanner. This allows for fast 3D scanning without motion-induced artefacts caused by stage scanning, and selective imaging of regions of interest. A $20 \mathrm{MHz}$ fibre tip transducer is used for acoustic detection, which allows a variety of sample holders to be used including common approaches such as multi-well plates and petri-dishes.
\end{abstract}

Keywords: MEMS, photoacoustic microscopy, fibre tip transducer

\section{INTRODUCTION}

With the ever-increasing interest in evaluating many biological processes in 3D environments instead of single-layer cells, the deep tissue imaging capabilities of optical microscopy techniques are becoming more popular. Out of the variety of techniques that exist to date, for example multi-photon microscopy [1], adaptive optics enhanced microscopy [2], or the move to near-infrared probes [3], photoacoustic microscopy (PAM) occupies a unique section as it moves the detection of the sample response from the optical to the acoustic domain [4]. This allows deeper imaging in scattering 3D samples due to a lower scattering cross-section of acoustic waves compared to optical ones.

Next to deeper imaging potential, PAM also has the advantage of allowing label-free imaging due to its signal dependence on optical absorption instead of fluorescence labels. As such the potential for quantitative spectroscopic studies similar to Raman microscopy is possible, with the added benefit of potential stronger molecule vibration bands for photoacoustic approaches leading to the potential of stronger recovered imaging signals.

PAM has been shown in a variety of configurations to date, focusing mostly on the imaging of blood vessels due to the strong endogenous contrast of hemoglobin at a commonly used laser excitation wavelength of $532 \mathrm{~nm}$. The demonstrated configurations range from endoscopic approaches, using miniature rotating mirrors or Microelectromechanical Systems (MEMS) mirrors and miniature ultrasound transducers [5], to large scale tomographic readout systems for whole animal investigations [6] or the combination of MEMS and large area scanning for histopathology imaging [7]. Further recent configurations show imaging systems with the move to longer wavelength laser sources [8] and the combination of UV and infrared excitation to increase the achievable resolution of the systems [9].

In this work, we will show the development of an all-optical PAM system including optical MEMS micromirror for beam steering and positioning and optical fibre tip transducer for ultrasound readout. We will demonstrate the contrast of the test system and its operation principle before adapting the setup for high-resolution focused imaging for sub-cellular resolution.

*mark.donnachie@strath.ac.uk; centreformicrosystemsandphotonics.eee.strath.ac.uk 


\section{MEMS PHOTOACOUSTIC MICROSCOPY SYSTEM DESIGN AND CHARACTERISATION}

\subsection{Optical setup}

The PAM setup currently in development is shown in Figure 1. A Q-switched frequency tripled Nd:YAG laser (Teem Photonics PNV-B) is used as an excitation source, having a pulse repetition rate of $1 \mathrm{kHz}$ and laser pulse durations of $300 \mathrm{ps}$. The output laser beam is attenuated and guided through a telescope to reduce the beam diameter to $0.32 \mathrm{~mm}$, tailored to fit onto the surface of the $1 \mathrm{~mm}$ diameter MEMS (Mirrorcle A7M10.2-1000AL) used for beam positioning and raster-scanning. The deflected laser beam is focused by a $30 \mathrm{~mm}$ focal length lens (Thorlabs AC127-030), with the lens creating a telecentric imaging field, and guided into a water bath. The laser focal spot size is measured at a full width half maximum of $23 \mu \mathrm{m}$. The test samples and phantoms are mounted on microscope slides and placed in the focus of the optical excitation. A fibre optic hydrophone (Precision Acoustic TFS) is placed $0.5 \mathrm{~mm}$ behind the sample at a $45^{\circ}$ angle to the imaging plane, acting as an ultrasound transducer. A $635 \mathrm{~nm}$ diode laser is collinearly aligned with the photoacoustic excitation laser and acts as a beam positioning element. The signal from the fibre tip sensor is demodulated and recovered using a USB DAQ (Picoscope 6404D). A fast photodiode is used as the trigger for data acquisition.

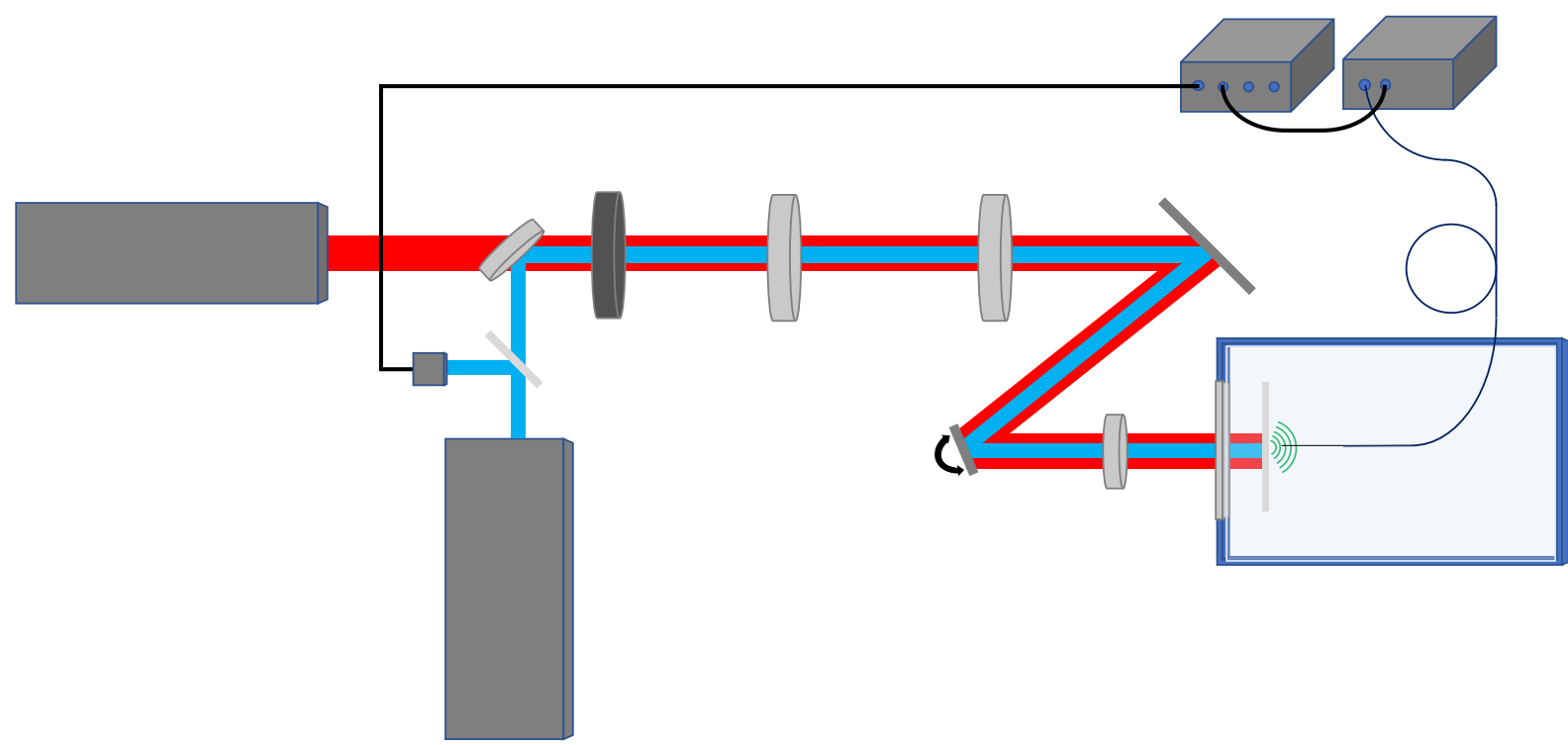

Figure 1. Schematic of MEMS photoacoustic microscopy system. The $355 \mathrm{~nm}$ excitation laser beam is shown in light blue, exciting a photoacoustic signal - shown in green - at the target. The $635 \mathrm{~nm}$ laser is shown in red and is used as a guide beam to aid with the alignment of the target and fibre optic hydrophone. The signal from the interrogation unit is captured with the oscilloscope which is triggered by the photodiode.

\subsection{MEMS and fibre tip transducer characterisation}

To control the laser positioning on the sample and create the raster-scan required for PAM image reconstruction a Mirrorcle MEMS micromirror with $1 \mathrm{~mm}$ mirror diameter is used. The 2-axis mirror allows for quasi-static movement in both axes, controlled through a dual-channel signal generator (Rigol DG1022Z) and an analogue driver board from Mirrorcle. The achievable static scan angles were characterised by reflecting a beam onto a screen and measuring the displacement at various input voltages, showing a maximum optical angle of $\pm 10^{\circ}$ in both axes (see Figure $2 \mathrm{~A}$ ). The dynamic behaviour of the mirror was characterized using a camera to capture the path traced by the displaced beam. The image was analyzed to find the maximum displacement at each frequency which is shown in Figure $2 \mathrm{~B}$. The quasi-static response drops off 
around $200 \mathrm{~Hz}$, with a resonance response visible at $4.52 \mathrm{kHz}$. The step-response of the device was measured using a quadrant photodiode and small-signal actuation of $10 \mathrm{mV}$, showing a response time of $0.6 \mathrm{~ms}$.
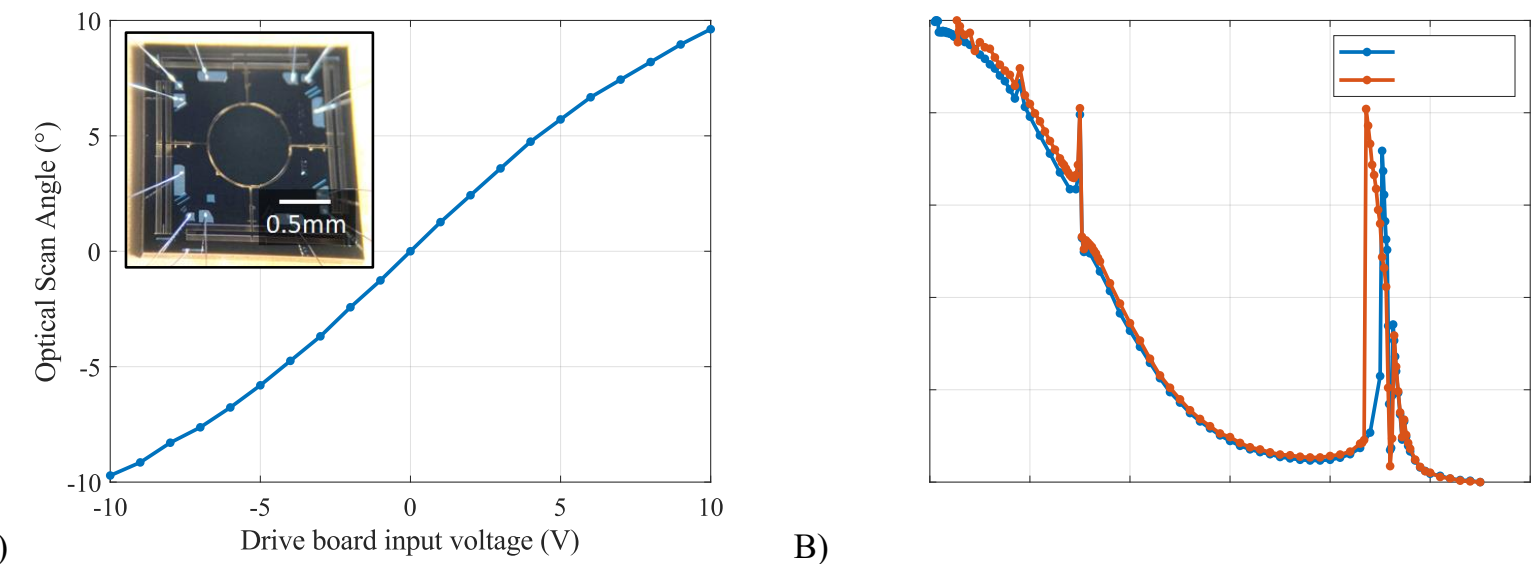

A)

Figure 2. A) Measured full optical scan angle of 2-axis MEMS with the device shown inset. B) Frequency response of 2-axis MEMS measured with a $\pm 3 \mathrm{~V}$ sine wave input, with traces for both ascending and descending voltages demonstrating hysteresis at around $4.5 \mathrm{kHz}$.

The frequency response of the fibre optic hydrophone was provided by the Precision Acoustics operating software of the respective read-put unit and is shown in Figure 3 with the peak sensitivity at $24 \mathrm{MHz}$.

The photoacoustic images were formed by recording the $1 \mathrm{kHz}$ repetition rate A-scans of the fibre optic hydrophone transducer with the oscilloscope. Each A-scan consists of 200 averages, with a laser pulse energy of $0.24 \mathrm{~J} / \mathrm{cm}^{2}$ per pulse at the sample. As the MEMS scans across the sample, each row of A-scans is formed into a 2D image depicting the depth vs beam position. After all the rows are captured, the images for each row are forming a 3D stack of images. A maximum intensity Z-projection is then performed to provide a 2D image of the target as seen in Figure 4 A and Figure $5 \mathrm{~A}$.

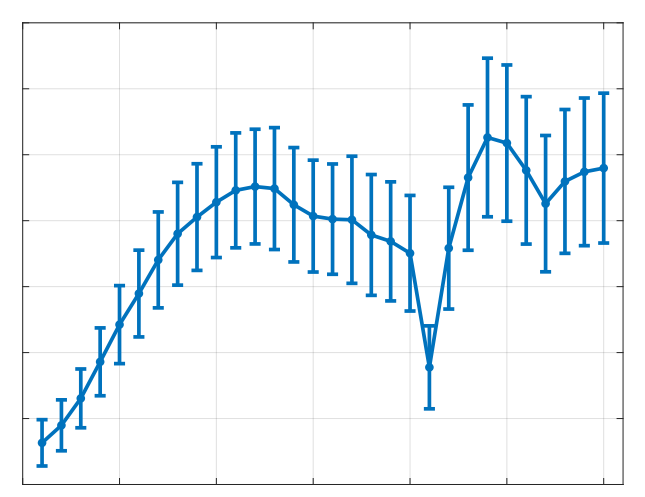

Figure 3. Frequency response of the fibre optic hydrophone ultrasound transducer. 


\section{RESULTS}

\subsection{USAF test target}

A Positive 1951 USAF Wheel Pattern Test Target (Thorlabs R3L1S4P) was used to characterize the system resolution. The target was placed in the water bath and positioned in the focal plane of the excitation beam and aligned relative to the fibre optic hydrophone. The sample was raster-scanned using the MEMS with a step-size of $2.9 \mu$ m. A maximum intensity projection image was produced from the captured A-scans and is shown in Figure 4 A. The region of the target scanned was element 1 of group 3, showing a line-width of $62.5 \mu \mathrm{m}$. A line profile across the line pairs is shown in Figure 4 B.

A)

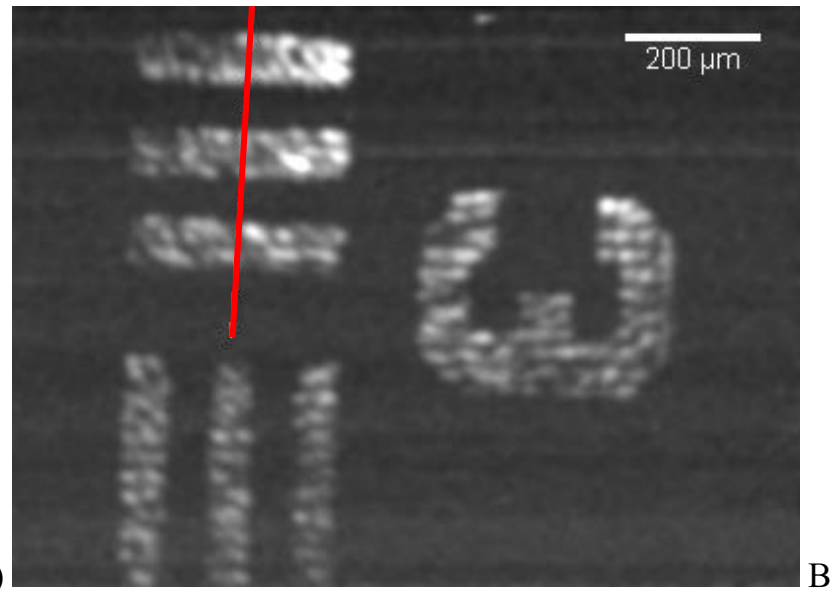

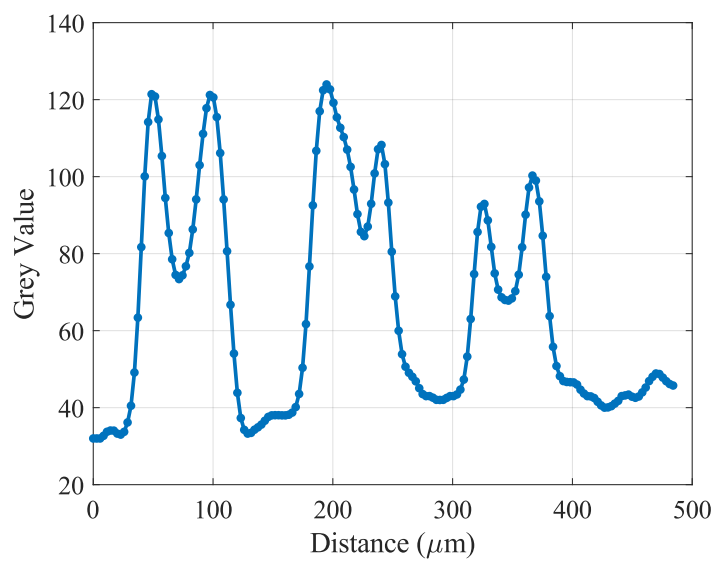

Figure 4. A) Photoacoustic image of 1951 USAF target group 3 element 1. To reduce noise, a Gaussian blur filter was applied during post-processing $(\sigma=1.75)$. B) Line profile of the red marked region in A.

A portion of a square target of the test pattern was also scanned to determine the system resolution and is shown in Figure 5 A. The red line in Figure 5 A shows where the line profile in Figure 5 B was taken. The derivative of the measured edge spread function was calculated and a Gaussian profile was fitted to it to determine the point-spread function of the system. This Gaussian is shown in orange in Figure 5 B. The FWHM was calculated to be $23.6 \mu \mathrm{m}$, indicating the lateral resolution of the system using the low-magnification $30 \mathrm{~mm}$ focal length lens for the laser excitation.

A)

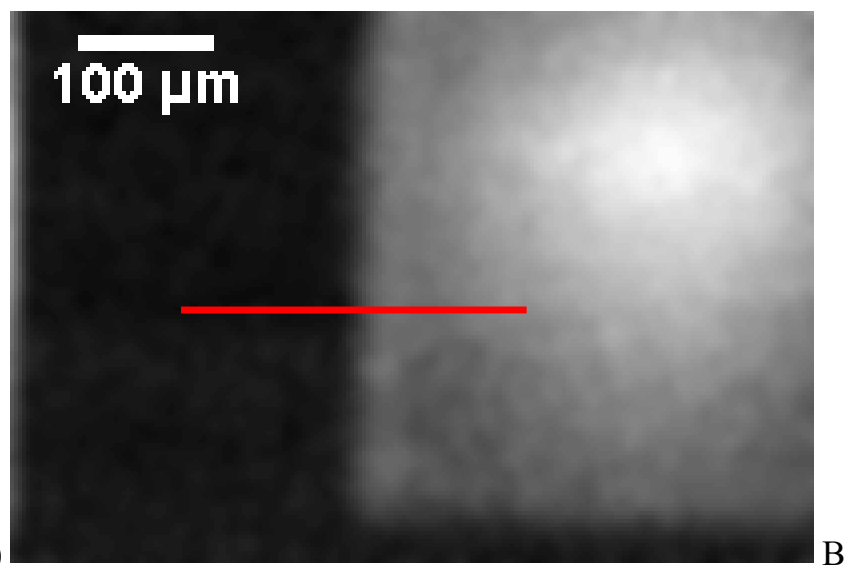

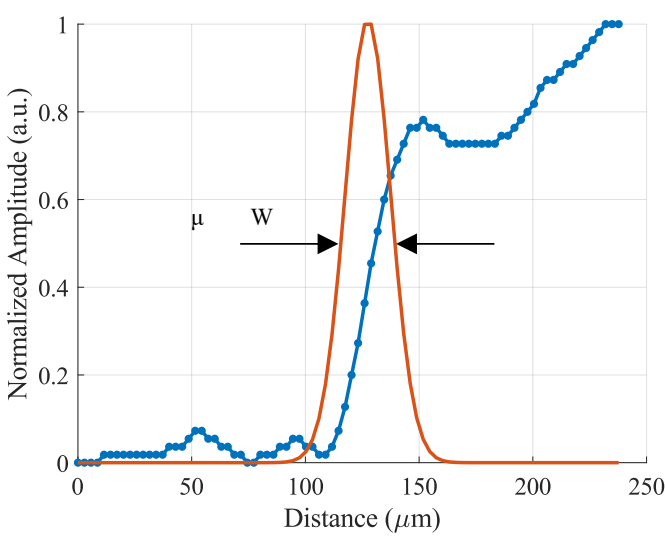

Figure 5. A) Photoacoustic image of the square region of the USAF target taken using the Mirrorcle MEMS device and the fibre optic hydrophone. B) Edge spread function, shown in blue, of the red line in A with the Gaussian fitted to the derivative of the edge spread function shown in orange. The FWHM is calculated to be $23.6 \mu \mathrm{m}$. 


\subsection{Phantom images}

A three-dimensional test sample was created using $7.5 \mu \mathrm{m}$ thick carbon fibres, creating a lattice with individual fibres overlapping in close proximity at crossover points. An example maximum intensity reconstruction using the PAM system is shown in Figure 6 A, with a comparison image using an optical microscope shown in Figure 6 B. The carbon fibres are clearly distinguishable, with their apparent width limited by the system resolution of $23 \mu \mathrm{m}$.
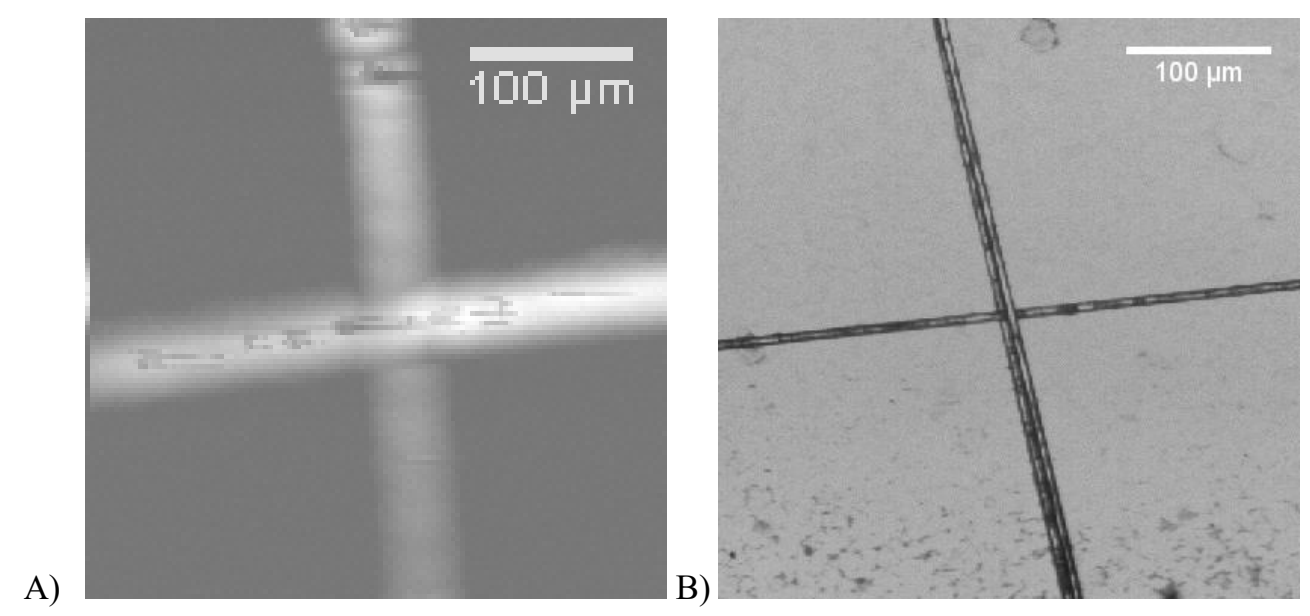

Figure 6. A) Photoacoustic image of crossed carbon fibres with a diameter of $7.5 \mu \mathrm{m}$. B) Image of the same carbon fibre cross captured with a microscope.

\section{CONCLUSION}

We have shown the design and characterization of an all-optical photoacoustic microscopy system, using a 2-axis MEMS micromirror and a fibre-tip optical transducer for image generation without sample movement. Both the MEMS and transducer have been characterized, showing a step response time below $1 \mathrm{~ms}$ for raster scanning and an acoustic centre frequency around $24 \mathrm{MHz}$. The system performance has been evaluated using microscope slide mounted samples, showing an in-plane resolution of $23 \mu \mathrm{m}$ and clear recovery of 3D objects.

\section{ACKNOWLEDGEMENT}

We acknowledge funding from the UK Engineering and Physical Sciences Research Council (grant EP/S032606/1, doctoral training partnership grant EP/R513349/1) and the UK Royal Academy of Engineering (Engineering for Development Fellowship scheme RF1516/15/8).

\section{REFERENCES}

[1] Miller, D. R., Jarrett, J. W., Hassan, A. M. and Dunn, A. K., "Deep tissue imaging with multiphoton fluorescence microscopy," Curr. Opin. Biomed. Eng. 4, 32-39 (2017). DOI: 10.1016/j.cobme.2017.09.004

[2] , X "Th -dimensional adaptive optical nanoscopy for thick specimen imaging at sub-50-

Nat. Methods 18, 688-693 (2021). DOI: 10.1038/s41592-021-01149-9

[3] Liu, P., Mu, X., Zhang, X. D. \& Min , "Th ～-Infrared-II Fluorophores and Advanced Microscopy

$\mathrm{T} h$

B "Bioconjug. Chem. 31, 260-275 (2020). DOI:

10.1021/acs.bioconjchem.9b00610 
[ ] J , , K , J, L , , B , J W \& K , C, "R w 15, 100141 (2019). DOI: 10.1016/j.pacs.2019.100141

[ ] G , , C , X , \& X, L, “ h 42, 4615 (2017). DOI: $10.1364 / \mathrm{ol} .42 .004615$

[ ] W , L \& , " h 62 (2012). DOI: $10.1126 /$ science. 1216210 [7] Baik, J W , "I L b Rev. 15,2100

h : $\mathrm{h}$ $\mathrm{h}$

b $\quad$ E

f

h $\quad f C$
,"Photoacoustics

, Opt. Lett.

," Science 335, 1458-

," Laser Photon.

(2021). DOI. $10.1002 /$ por.202100124

,$" J$

[ ] , Y , "L b -free imaging of lipid-rich biological tissues by mid-infrared photo

Biomed. Opt. 25, 1-7 (2020). DOI: 10.1117/1.jbo.25.10.106506

[9] h, J , " h -resolution, high-contrast mid-infrared imaging of fresh biological samples with ultraviolet- 